\title{
Treatment of myasthenia gravis with adrenocorticotropic hormone (ACTH): massive short-term and maintenance treatment ${ }^{1}$
}

\author{
CHARLES A. CAPE AND ROBERT A. UTTERBACK \\ From the Department of Neurology, University of Tennessee College of Medicine, Memphis, \\ Tennessee, U.S.A.
}

The management of severe myasthenia gravis presents a continuing challenge to the physician. Even with the most careful adjustment of anticholinesterase medication the patient may become severely disabled. Thymectomy seems to offer real benefit to some of the carefully selected patients. However, despite the careful employment of anticholinesterase medication and the improvement after some thymectomies, some patients still cannot be adequately controlled and become severely disabled and die.

In 1951 Torda and Wolff treated these refractory myasthenia patients with ACTH. They obtained a 'significant partial remission' after corticotropin therapy in 10 of 15 patients. They recognized, however, that a transient and sometimes severe exacerbation of weakness was apt to occur during the first days of treatment. One of their 15 patients died during such an exacerbation. Their report was directly contradicted by Grob and Harvey, who reported in 1952 that in none of their 10 patients was any increase in strength observed either during or after ACTH administration.

In subsequent years, however, there have been additional reports (Grob and Namba, 1966; Osserman and Genkins, 1966; von Reis, Liljestrand, and Matell, 1966) of remissions lasting three months or longer. Such remissions were more consistent after massive doses of ACTH. von Reis et al. (1966), for example, gave $1,000 \mathrm{u}$. ACTH over a period of six to 12 days to 13 patients and obtained excellent results in 23 out of 33 courses of treatment.

Ample clinical experience has shown that massive doses of ACTH will often bring about a remission when myasthenia is otherwise refractory to medication. The reports have uniformly noted that initiation of treatment carries danger because of transient increase of weakness, and all have lamented the brevity of the remission which might

'This paper was read at the meeting of the Memphis Neurological Society, Memphis, Tennessee, 8 June, 1968. last only a few weeks. Unfortunately, this remission may be followed by another exacerbation. Osserman and Genkins in 1966 commented that in one of their patients 'clinical improvement ... has been maintained by serial weekly and biweekly injections of corticotropin that are given on an out-patient basis.' We have attempted to prolong remissions in our patients by continuing maintenance therapy and have been surprised by the favourable results 0 The present study relates our experience with the beneficial effect of massive doses of ACTH in five patients with refractory myasthenia gravis, and wit maintenance therapy with ACTH in three patients for 16 to 23 months.

CASE REPORTS

CASE 1 A 24-year-old girl had myasthenia gravis, group III, according to the clinical classification of myasthenia gravis (Table I). She had numerous crises requiring emergency tracheostomies and continuous respiratory assistance; eventually she was left with a permanent tracheostomy. Her disease was never adequately controlled on anticholinesterase medication, and for long periods she was scarcely able to survive. Neurological examination during this period showed bilateral ptosis with external ophthalmoplegia and bilateral facial paresis. The palate elevated very poorly and she was dysarthric. The strength of the muscles of the upper and lower extremities was grade 3 (Medical Research Council, 1942). She was confined to her house because of her poor physical reserve. On 21 June 1965, she was started on a 10-day course of ACTH, receiving $100 \mathrm{u}$. per day intramuscularly for a total of $1,000 \mathrm{u}$. She began a dramatic improvement on 22 June 1965 without any prior worsening of her myasthenic symptoms. She did not require anticholinesterase medication during most of the three-month remission. Examination during this remission showed residual external ophthalmoplegia of the left eye except for downward gaze, but there were nearly normal movements of the right eye. There was no ptosis or facial paresis. The strength of her extremities had increased to greater than grade 4 and her exercise tolerance allowed her to assume nearly normal activities. 
TABLE I

CLINICAL CLASSIFICATION OF MYASTHENIA GRAVIS IN ADULTS ${ }^{1}$

Group I: Ocular

Involvement of single muscle group; mostly ocular, appearing as ptosis and diplopia. Very mild; no mortality

Group IIA: Mild generalized

Gradual onset; frequently presents with ocular symptoms spreading to generalized involvement of skeletal and bulbar musculature. Respiratory system not involved. Usually mild; very low mortality

Group IIB: Moderate generalized

Gradual onset. Group includes ocular involvement with moderately severe skeletal and bulbar involvement. Respiratory system not involved. Low mortality

Group III: Acute fulminating

Rapid onset of severe bulbar and skeletal muscle weakness with early involvement of respiration. Very severe; high mortality

Group IV: Late severe

Exacerbation of disease in patients of groups I or II approximately two years after onset of disease. Symptoms and course similar to group III

${ }^{1}$ From Osserman and Genkins (1966).

A gradual decrease in strength was accentuated by a severe exacerbation. By December 1965 the strength of the extremity muscles was down to grade 3 . In addition to the external ophthalmoplegia of the left eye there was again impaired upward gaze of the right eye and bilateral ptosis. A second course of 1,000 u. ACTH was started on 8 December. On the fourth day of treatment, 11 December, she became considerably weaker and required respiratory support. On 12 December she was unable to swallow, and the strength of her extremity muscles had decreased to grade 2 . She continued to require respiratory assistance until 15 December, when she began improving. During the ensuing remission she had normal respirations and deglutition, but there was no change in her ophthalmoplegia. Her extremity strength improved to grade 4. This improvement lasted only 33 days. Beginning 10 January 1966, an additional $200 \mathrm{u}$. ACTH were given over five days, with no improvement.

Even though radiographs of the anterior mediastinum did not reveal an enlarged thymus, a thymectomy was performed on 21 March 1966. She was found to have an enlarged thymus, with some portions showing 'hyperplasis'. This was followed by a remission lasting two months with her extremity strength improving to grade 4 .

By December 1966 her strength had decreased to grade 3 , and the ophthalmoplegia was worse. It was clear the thymectomy had not produced a lasting remission. Her strength was so poor that a final course of $800 \mathrm{u}$. ACTH was given that month, followed again by a remission. During this intensive course of ACTH there was no significant worsening of her symptoms; she had some improvement in the ophthalmoplegia and in the facial paresis, and the strength of her extremities improved to grade 4 . She had a moderate improvement in her exercise tolerances.

When her strength began to fail once more (extremity strength grade 3), she was started on weekly injections of $100 \mathrm{u}$. ACTH. Her strength gradually improved over the next few months. In the muscles of her upper and lower extremities the strength increased to greater than grade 4, and has remained so for 23 months. She does not have ptosis, respiratory or swallowing difficulties, but she still has paresis of the ocular muscles on the left. Where she was previously confined to her home, she is now leading a more active life. She has enrolled as a part-time student at the university, resuming her education which was interrupted by the severe myasthenia.

In summary, this young lady was totally disabled and frequently bedfast in spite of a thymectomy and intensive therapy with anticholinesterase medication. Intensive courses of ACTH were consistently followed by improvements in her strength, but these had been regularly followed by recurrences of the weakness until she was started on maintenance ACTH. With maintenance ACTH injections of $100 \mathrm{u}$. weekly her strength has remained improved; there has been no recurrence of the weakness for a period of 23 months. She continues to use Mestinon (pyridostigmine), $60 \mathrm{mg}$ three to five times daily.

CASE 2 A 51-year-old Negro male had the onset of severe generalized and bulbar myasthenia gravis (group III) in December 1965. Despite the benefit derived from anticholinesterase medication he was always in moderate respiratory distress and often was confined to bed during the next nine months. His anticholinesterase medications were carefully regulated in the hospital. On what was considered optimal dosage of Mytelase (ambenonium) his neurological examination showed severe bilateral ptosis, inability to converge or to adduct either eye, and paresis of abduction resulting in nystagmoid jerks. He was unable to gaze either upward or downward. There was mild bilateral facial paresis. The strength of the proximal muscles of the upper extremities was grade 4 and that of the distal muscles was grade 3 . The strength of the proximal muscles of the lower extremities was grade 3 and that of the distal muscles was grade 2 . $\mathrm{He}$ went into a myasthenic crisis when one dose of his medication was skipped.

After nine months of fruitless efforts to control his myasthenia with anticholinesterase medication, he was given 1,040 u. ACTH intramuscularly over eight days beginning 18 October 1966 . He became weaker and required continuous automatic respiratory support in the intensive care unit until 24 October. During this period his strength was less than grade 1. It began to improve rapidly on 24 October, and by 27 October he had no 
demonstrable weakness (grade 5). He stated that he felt as healthy as he had felt before his illness. This remission continued until December 1966, when he developed extraocular muscle weakness and weakness of the distal muscles of the upper and lower extremities (grade 4). The muscles innervated by the other cranial nerves, and the proximal extremity muscles had normal strength (grade 5).

In December 1966 he was started on sporadic injections of $100 \mathrm{u}$. ACTH. Because of the distance he lived from the University of Tennessee, he was not treated with regular weekly injections of ACTH. There was no further increase in his weakness. Attempts to improve his strength with various doses of anticholinesterase medication produced no significant change. All anticholinesterase was discontinued in March 1967 with no change in his strength. He has been taking 30 to $120 \mathrm{mg}$ Mestinon a day since February 1968 with very minimal effects on his strength.

A neurological examination in January 1969 showed persistent bilateral ptosis and inability to adduct either eye or to converge his eyes, but the upward and downward movements were normal. There was no facial paresis, dysphagia, or dysarthria. The strength in the proximal muscles of the extremities was grade 5 , whereas strength of the distal muscles was grade 4 .

In summary, he obtained a complete remission from an intensive course of ACTH and he has maintained most of this improvement on periodic injections of ACTH. Supplementary therapy with anticholinesterase medication has not seemed to alter his strength appreciably.

CASE 3 A 69-year-old man had the onset of bulbar myasthenia gravis (group IIA), in March 1963. Initially the disease was confined to the ocular muscles, but subsequently he developed hoarseness, dysphagia, and difficulty in chewing. In spite of increasing dosage of Mestinon these symptoms could not be adequately controlled. Neurological examination performed while he was on optimal dosage of medication showed bilateral ptosis, external ophthalmoplegia, mild bilateral facial paresis, dysphagia, and difficulty in chewing for more than four or five bites. There was no weakness of the extremity muscles.

ACTH treatment was initiated on 21 November 1966 and he was given $700 \mathrm{u}$. over eight days. The following day he felt weak and dizzy, but he did not have any other complications of the ACTH treatment. His myasthenia remained unchanged until two days after his last dose of ACTH, when there was an obvious improvement in his bulbar symptoms. During the next 60 days he had a persistent minimal disturbance of conjugate gaze, but no specific ocular paresis. There was no ptosis, facial weakness, dysarthria, dysphagia, or impairment of his chewing.

Unfortunately, all of his symptoms gradually recurred in spite of careful adjustments of his medications. By the end of February 1967 the neurological findings were very similar to those recorded before the first course of ACTH; his chewing and swallowing were significantly worse. A second course of ACTH, $100 \mathrm{u}$. a day for 10 days, was started on 2 March 1967. He felt subjectively weaker toward the end of this course of treatment, but the results of neurological examination were unchanged until the last day of injections, 11 March, when he became dyspnoeic and his dysphagia and dysarthria became worse. This exacerbation of his myasthenia lasted five days and he then had a remission lasting $\mathbf{4 0}$ days. During this period he was asymptomatic except for mild weakness of the ocular muscles.

His myasthenia recurred, and again was refractory to treatment with anticholinesterase medication. The neurological findings were essentially the same as they had been before the second course of ACTH. One thousand units ACTH were given over a period of 10 days beginning 19 May 1967. On the second day of injections he became very weak, dyspnoeic, and could not swallow, but this was followed promptly by a remission which lasted over two months. During this remission, as with the other remissions, he continued taking 60 to $90 \mathrm{mg}$ Mestinon four times a day.

Early in September his symptoms were beginning to be troublesome once more. Examination at this time revealed almost complete external ophthalmoplegia; there was minimal lateral rectus movement bilaterally. He had bilateral ptosis and facial weakness, but no dysphagia. Although this did not represent so complete an exacerbation as he had previously suffered, we decided to offer another course of intensive therapy and follow it with maintenance ACTH treatment. On 11 September 1967, the fourth course of $1,000 \mathrm{u}$. was started. He developed generalized weakness of the extremities (grade 4) on the fourth, fifth, and sixth days of ACTH treatment, but by the seventh day this began to clear. There was then a rapid remission of all his symptoms; soon there was absolutely no paresis of any of the ocular or bulbar muscles.

After this fourth intensive course of ACTH he was maintained on 100 u. ACTH intramuscularly every week. There was no recurrence of any weakness, either subjectively or by examination, for the next three months. During December 1967 he developed a mild paresis of his left medial rectus muscle, along with a barely detectable ptosis on that side. This ptosis gradually disappeared, but a very mild paresis of the left medial rectus was still evident in January 1969.

In summary, this patient obtained complete remissions after each of three courses of intensive ACTH therapy. Each of these remissions was followed by another exacerbation within two months. After a fourth intensive course he was given weekly injections of $\mathrm{ACTH}$, and the remission has been maintained continuously for 16 months.

CASE 4 A 24-year-old housewife had the onset of acute fulminating myasthenia gravis (group III) in December 1966. She noticed ptosis of the left eyelid about 1 December 1966. Subsequently she developed diplopia, dysarthria, dysphagia, and weakness of the upper and lower extremities. Increasing doses of Mestinon, and then Mytelase, failed to control her myasthenia, so she was referred to the University of Tennessee. The diagnosis of myasthenia gravis was confirmed, and a chest radiograph revealed a $5 \times 7 \mathrm{~cm}$ mass in the anterior mediastinum. A thymoma of lymphocytic type was almost completely removed on 24 February 1967 . The thymoma 
engulfed the left phrenic nerve and the recurrent branch of the vagus nerve. Surgery was followed by radiation therapy to the mediastinum. She got fair relief from her weakness on $360 \mathrm{mg}$ Mestinon a day for three weeks post-operatively, but then became weaker in spite of increasing doses of Mestinon. She was still poorly controlled several weeks later when the dose had been increased to $240 \mathrm{mg}$ five times a day. Neurological examination at a time when she was under optimal control with this medication showed ptosis of the left eyelid, weakness of the masseter and pterygoid muscles, and bilateral facial paresis. The sternocleidomastoid, trapezius, and extensor muscles of the neck were grade 3 . The strength of the shoulder and pelvic girdle muscles was grade 3 and that of the distal extremity muscles was grade 4. Mestinon was decreased before ACTH treatment to eliminate the possibility of over-medication.

Beginning 15 May 1967 she received 1,000 u. ACTH over 10 days. She became abruptly weaker on the seventh day of treatment, with an accentuation of her difficulty in chewing and swallowing. In addition she was unable to raise her arms above her head or rise from a chair. The accentuation of her weakness was temporary, and by 24 May she began improving. By 20 June 1967 she was asymptomatic and her neurological examination was completely normal except for fatigue with repetitive muscle contraction. There was no ptosis, facial paresis, or weakness of the masseter muscles. The strength of the neck muscles and the girdle and extremity muscles was grade 5. She was taking 120 to $150 \mathrm{mg}$ Mestinon five times a day.

Maintenance ACTH in a dose of $100 \mathrm{u}$. per week was given every week or every other week after the intensive course of ACTH. She has remained asymptomatic and her muscles do not fatigue with repetitive contraction. She has gradually decreased her Mestinon to $15 \mathrm{mg}$ twice a day.

During February 1968 she developed impairment of temperature sensation in the right leg. Neurological examination confirmed this impairment, and also revealed more active tendon reflexes in the left leg and absent abdominal reflexes on the left. The neurological examination was otherwise normal. These changes were suspected to be manifestations of radiation myelopathy. A chest radiograph taken in March 1968 showed a recurrence of the anterior mediastinal mass. There has been no further increase in size of the mediastinal mass and no recurrence of the myasthenia.

In summary, this 24-year-old female did not obtain significant remission from her myasthenia when her thymoma was excised. An intensive course of ACTH did produce a remission. This remission has been maintained for 20 months by weekly injections of ACTH. The remission has persisted in spite of radiographic evidence of recurrence of her thymoma.

CASE 5 A 66-year-old lady had the onset of skeletal and bulbar myasthenia gravis (group IIB) in December 1966. Her symptoms did not respond well to anticholinesterase medications. While on optimal neostigmine treatment she continued to have severe external ophthalmoplegia. She could elevate both eyes only $10^{\circ}$, depress them $30^{\circ}$, and adduct them $10^{\circ}$. The right eye would adduct $30^{\circ}$ and the left $10^{\circ}$. She had bilateral facial weakness. The strength of the muscles of mastication was grade 3, and with minimal use they would quickly fatigue to grade 2 . She had moderate dysphagia and dysarthria. The strength of the neck flexors and extensors was grade 4 . Her other skeletal muscles had normal strength, but they would fatigue rapidly. She was hardly able to walk around the bed before she was overcome with severe weakness, which was sometimes associated with respiratory distress. Any attempt to reduce her neostigmine dosage promptly produced severe weakness and respiratory disease.

A course of 920 u. ACTH intramuscularly was given between 25 July 1967 and 3 August 1967 . On the fourth day of treatment she had an accentuation of her symptoms -more difficulty in swallowing, chewing, talking, and an increase in her extremity weakness. She became apnoeic and on a few occasions required respiratory assistance. Her ACTH treatment was complicated by cystitis and thrombophlebitis. On 6 August 1967 she began improving, and by 9 August she had minimal ocular paresis and no facial weakness. Deglutition and speech were normal. She could chew solid food and could eat all of her meals without difficulty. There was no weakness of the neck muscles. She could walk in the hospital corridors without fatigue. This remission continued until at least the first week of October 1967. A neurological examination during September was entirely normal except for impaired conjugate gaze to the right; there was no specific ocular muscle paresis. She still gave a history of decreased exercise tolerance. She was not given maintenance therapy and did not continue in our care. According to her local physician she then became weaker during October and did not respond to increased dosage of anticholinesterase medication. As a last resort he hospitalized her in December 1967 and began treating her with $100 \mathrm{u}$. ACTH intramuscularly daily. On the fifth day of treatment she became unresponsive, and she died on the sixth day. Necropsy was not permitted.

In summary, this elderly lady was completely disabled by myasthenia in spite of anticholinesterase medication. Intensive ACTH therapy produced a two-month remission. She was not given maintenance therapy. On a second trial of intensive ACTH therapy she suddenly grew worse and died.

\section{RESULTS}

A diagnosis of myasthenia gravis was definitely established in all of the patients by the classical history and physical findings, by the improvement in strength after anticholinesterase medication, and/ or by the decline in amplitude of muscle action potentials after repetitive nerve stimulation. All of the patients had moderate to severe myasthenia that could not be adequately controlled with anticholinesterase medication. Two of the patients had thymectomies followed by brief remissions. This had no apparent influence on the ACTH therapy.

Corticotropin therapy resulted in significant improvement in all of our patients (Table II). One 
TABLE II

RESULTS OF ACTH THERAPY

\begin{tabular}{|c|c|c|c|c|c|c|c|}
\hline Case & Age & $\operatorname{Sex}$ & $\begin{array}{l}\text { Duration of } \\
\text { myasthenia } \\
\quad(\text { mth })\end{array}$ & $\begin{array}{l}\text { Duration of } \\
\text { improvement } \\
\text { (days) }\end{array}$ & $\begin{array}{l}\text { Clinical } \\
\text { evaluation of } \\
\text { results }\end{array}$ & Thymectomy & $\begin{array}{c}\text { Maintenance } \\
\text { ACTH; } \\
\text { duration of } \\
\text { improvement }(\mathrm{mth})\end{array}$ \\
\hline 2 & $\begin{array}{l}23 \\
23 \\
24 \\
51\end{array}$ & $\mathbf{M}$ & $\begin{array}{l}75 \\
80 \\
92 \\
11\end{array}$ & $\begin{array}{c}100 \\
33 \\
53 \\
42^{1} \\
750^{2}\end{array}$ & $\begin{array}{l}\text { Excellent } \\
\text { Fair } \\
\text { Good } \\
\text { Excellent } \\
\text { Excellent }\end{array}$ & 2 Mar. 66 & $\begin{array}{l}\text { Yes } 23 \\
\text { Sporadic }\end{array}$ \\
\hline 3 & $\begin{array}{l}68 \\
69 \\
69 \\
69\end{array}$ & $\mathbf{M}$ & $\begin{array}{l}44 \\
47 \\
50 \\
54\end{array}$ & $\begin{array}{c}60 \\
40 \\
63 \\
\text { Maintenance therapy }\end{array}$ & $\begin{array}{l}\text { Excellent } \\
\text { Excellent } \\
\text { Excellent } \\
\text { Excellent }\end{array}$ & & Yes 16 \\
\hline 4 & 24 & $\mathbf{F}$ & 6 & Maintenance therapy & Excellent & 24 Feb. 67 & Yes 20 \\
\hline 5 & $\begin{array}{l}66 \\
66\end{array}$ & $\begin{array}{l}\mathbf{F} \\
\mathbf{F}\end{array}$ & $\begin{array}{r}9 \\
13\end{array}$ & $\begin{array}{r}62 \\
0\end{array}$ & $\begin{array}{l}\text { Good } \\
\text { Died }\end{array}$ & & \\
\hline
\end{tabular}

${ }^{1}$ Extraocular muscles.

${ }^{2}$ All muscles excluding the extraocular muscles and the distal extremity muscles.

patient was given a second course of ACTH treatment when she was in a terminal state and she died on the sixth day of treatment. This course of ACTH was not administered by us so we did not include it in our series. Ten courses of ACTH were given in five patients. According to our clinical evaluation, seven of these courses of ACTH were followed by 'excellent' improvement in the patient's strength. Because the patients required anticholinesterase medication to be symptom free, we did not designate the improvement as a 'remission'. Two courses of ACTH were followed by 'good' clinical improvement. Improvement classified as 'fair' occurred after the remaining course of ACTH therapy. The difference between a good and fair response is based on the improvement in strength, the increase in exercise tolerance, and the improvement in the patient's general well-being.

The amount of ACTH we administered during intensive therapy varied from 600 to $1,040 \mathrm{u}$. The route of administration was always intramuscular. There was no apparent difference in the degree or the duration of improvement with these varying amounts of ACTH. There was a mild decrease in the patient's strength at some time during seven of the courses of ACTH treatment. This deterioration occurred between the first and tenth day of treatment. One patient had a severe accentuation of the myasthenic symptoms during treatment. The presence of an initial worsening, or the degree of the increased weakness, did not have any relationship to the subsequent improvement. On one occasion case 1 received $200 \mathrm{u}$. ACTH over a period of five days with no change in her symptoms. This was an inadequate dose (Grob and Namba, 1966), so we did not include it in our series.
Three of the five patients have been treated with weekly or twice-monthly injections of $100 \mathrm{u}$. ACTH. This has in each case been instituted after one or more intensive courses of ACTH therapy. On this maintenance therapy, all three have maintained 'good' to 'excellent' improvement-23 months, 16 months, and 20 months respectively.

\section{DISCUSSION}

In 1935, Simon empirically treated two cases o myasthenia gravis with anterior pituitary extract The results were very encouraging. Subsequent experiments by Torda and Wolff $(1944 a, b, c)$ suggested that ACTH augments the synthesis of acetylcholine and that the sera, cerebrospinal fluid, and thymic tissue from myasthenic patients inhibit acetylcholine synthesis. They believed there was a great enough relationship between ACTH and myasthenia gravis to warrant a clinical trial. They administered $400 \mathrm{mg}$ ACTH over five days to five patients with myasthenia gravis (Torda and Wolff, 1949). There was an initial worsening of the myasthenia until the second day after the treatment, at which time the patients began improving. An incomplete remission continued for three months. In 1951, the same authors reported the results of 15 patients treated with ACTH (500 mg over five days). A significant improvement occurred in 10 patients, no improvement occurred in four patients, and one patient died. They obtained more improvement in some patients with a second and a third series of ACTH. Millikan and Eaton (1951), at the Mayo Clinic, treated three myasthenic patients with ACTH. Excellent improvement occurred in one patient, mild improvement in another patient, and 
no improvement in the third patient. However, they treated two patients with cortisone and neither patient improved. Combining the patients treated with ACTH and cortisone their clinical trial did not show as favourable an improvement as Torda and Wolff's series. In 1952, Grob and Harvey treated 10 patients with ACTH and concluded that there was no significant improvement in any of the patients.

In 1966 von Reis et al. employed intensive shortterm courses of ACTH in severe myasthenia gravis with excellent results. They administered the ACTH subcutaneously in divided doses, starting with $180 \mathrm{u}$. per day and reducing it so that by six to 12 days approximately 1,000 i.u. were given during each course of therapy. Thirty-three courses of treatment were given to 13 severe cases of myasthenia gravis. There was excellent improvement after 23 courses, slight to moderate improvement after eight courses, and no improvement after two courses of treatment. The improvement was brief, with the average duration of the remissions in the patients having excellent improvement being seven months. Six patients who had relapses were given repeat courses of ACTH with more improvement after subsequent courses of treatment. These dramatic results encouraged the large myasthenia gravis centres to re-evaluate ACTH therapy. Osserman and Genkins (1966) confirmed von Reis and his colleagues' (1966) observations. Single courses of approximately $1,000 \mathrm{u}$. corticotropin were given intramuscularly to six patients. Four patients obtained significant benefit, one patient with a malignant thymoma obtained a good response, and the other patient, who had an unresectable thymoma, showed no improvement. Multiple courses of treatment in five patients resulted in excellent improvement in two patients, excellent improvement except for persisting dysphagia in one patient, and diminishing results after each course of treatment in two patients. The duration of improvement averaged three to four months. Grob and Namba (1966) evaluated the effect of a larger dosage of ACTH than they had used in their 1952 study and obtained gratifying results. They administered intravenously $1,000 \mathrm{u}$. ACTH over 10 days in eight patients with generalized myasthenia gravis. There was improvement in the patient's strength after 19 out of the 22 courses of corticotropin. No improvement occurred in the two patients who had the mildest myasthenia gravis. In addition, they administered $80 \mathrm{u}$. daily for only five days to nine patients with moderate to severe generalized myasthenia gravis with no subsequent improvement. This confirmed their previous experience with small dosages.

The great drawback of ACTH treatment appears to be the relatively short duration of improvement.
This has necessitated repeated courses in some patients. Osserman and Genkins (1966) have used maintenance therapy in one elderly male for six months. This patient received an intensive course of therapy followed in one week by a second intensive course of ACTH. Clinical improvement was maintained by weekly or bi-weekly injections of ACTH. The follow-up was too brief to conclude that the maintenance injections actually prolonged the remission that he would have received from the two intensive courses. We have treated three patients with $100 \mathrm{u}$. ACTH intramuscularly every one to two weeks. Two patients have maintained their remissions for 16 and 20 months after an intensive course of ACTH. The other patient's (case 1) weakness had partially recurred before maintenance therapy was initiated. Clinical improvement was induced by weekly injections of ACTH and has been maintained for 23 months. In spite of these encouraging results, the duration of maintenance therapy has been too brief to warrant any firm conclusion.

In conclusion, we want to emphasize the necessity of careful patient selection, of using adequate doses of ACTH, and of 'compulsive' supervision of the patients in intensive medical care units during the treatment. Deviation from these guidelines will result in either poor results or death. In addition, we wish to call attention to the possibility that 'remissions' may be prolonged by maintenance therapy with ACTH injected weekly or less often.

\section{SUMMARY}

Five patients with myasthenia gravis have been treated with brief, massive doses of ACTH and three of these patients have also been given maintenance therapy. A significant improvement occurred after intensive ACTH therapy in all patients, but the benefit lasted an average of only 57 days. Maintenance therapy has prolonged the improvement, but the duration of treatment has been brief.

\section{REFERENCES}

Grob, D., and Harvey, A. M. (1952). Effect of adrenocorticotropic hormone (ACTH) and cortisone administration in patients with myasthenia gravis and report of onset of myasthenia gravis during prolonged cortisone administration. Bull. Johns Hopk. Hosp., 91, 124-136.

- and Namba, T. (1966). Corticotropin in generalized myasthenia gravis. Effect of short, intensive courses. J. Amer. med. Ass., 198, 703-707.

Medical Research Council. (1942). Aids to the Investigation of Peripheral Nerve Injuries. M.R.C.(War) Memor. No. 7. H.M.S.O., London.

Millikan, C. H., and Eaton, L. M. (1951). Clinical evaluation of ACTH and cortisone in myasthenia gravis. Neurology (Minneap.), 1, 145-152. 
Osserman, K. E., and Genkins, G. (1966). Studies in myasthenia gravis. Short-term massive corticotropin therapy. J. Amer. med. Ass., 198, 699-702.

Simon, H. E. (1935). Myasthenia gravis; effect of treatment with anterior pituitary extract; Preliminary report. Ibid., 104, 2065-2066.

Torda, C., and Wolff, H. G. (1944a). Effect of adrenotrophic hormone of pituitary gland on ability of tissue to synthesize acetylcholine. Proc. Soc. exp. Biol. (N.Y.), 57, 137-139.

- - (1944b). Effect of blood serum from patients with myasthenia gravis on synthesis of acetylcholine in vitro. J. Clin. Invest., 23, 649-656. (1944c). Effect of spinal fluid from patients with myasthenia gravis on the synthesis of acetylcholine in vitro. Science, 100, 200-201.

(1949). Effects of adrenocorticotrophic hormone on neuro-muscular function in patients with myasthenia gravis. $J$. clin. Invest., 28, 1288-1235.

(1951). Effects of administration of the adrenocorticotropic hormone (ACTH) on patients with myasthenia gravis. Arch. Neurol. Psychiat. (Chic.), 66, 163-170.

von Reis, G., Liljestrand, A., and Matell, G. (1966). Treatment of severe myasthenia gravis with large doses of ACTH. Ann. N.Y. Acad. Sci., 135, 409-416. 